ISSN 0103-5150

Fisioter. Mov., Curitiba, v. 28, n. 2, p. 383-394, Apr./June 2015

Licenciado sob uma Licença Creative Commons DOI: http://dx.doi.org.10.1590/0103-5150.028.002.A019

\title{
Efeito agudo do alongamento na marcha de idosas em terreno inclinado
}

\section{Acute effects of stretching on elderly gait on inclined surface}

\author{
Ricardo Martins de Souza ${ }^{[a]}$, Bianca Kirchner ${ }^{[a]}$, André Luiz Félix Rodacki ${ }^{[b] *}$ \\ [a] UniBrasil Centro Universitário, Centro de Pesquisa em Atividade Física, Exercício e Esporte (CEPAFEE), Curitiba, PR, Brazil \\ [b] Universidade Federal do Paraná (UFPR), Setor de Ciências Biológicas, Departamento de Educação Física, Curitiba, PR, Brazil
}

\section{Resumo}

Introdução: 0 envelhecimento causa modificações musculares que influenciam negativamente a capacidade funcional dos idosos na locomoção, em especial durante a transposição de superfícies inclinadas. Essas mudanças geram redução da performance, diminuindo a mobilidade e aumentando o risco de queda na execução de tal tarefa. Os exercícios de alongamento podem reduzir a influência de dessas degenerações, preservando a capacidade muscular e a mobilidade. Objetivo: 0 objetivo deste estudo foi avaliar a atuação do efeito agudo de uma sessão de exercícios de alongamento sobre a marcha de idosas durante a locomoção em uma rampa. Materiais e métodos: Doze mulheres idosas, saudáveis e independentes, caminharam subindo e descendo uma rampa com inclinação de 10\%. A análise cinemática (2D) e eletromiográfica foi realizada durante uma única sessão experimental, imediatamente antes (PRÉ) e após (PÓS) a realização de um exercício de alongamento estático dos flexores do quadril. Resultados: 0 exercício de alongamento gerou aumento da amplitude (PRÉ $52,3 \pm 18,3^{\circ}$; PÓS 63,6 \pm 16,90) e velocidade (PRÉ 195,8 $\pm 31,2^{\circ}$ /s; PÓS $241,8 \pm 29,8^{\circ} /$ s) do quadril, assim como redução do tempo de ativação do tibial anterior

* RMS: doutor, e-mail: profricardo2006@yahoo.com.br BK: graduada, e-mail: biancakirchner@yahoo.com.br ALFR: doutor, e-mail: rodacki@ufpr.br 
(PRÉ TAFIN1 29,7 \pm 6,7\%; TAFIN2 100,0 \pm 0,0\%; PÓS TAFIN1 23,3 \pm 7,4\%; TAFIN2 87,7 $\pm 3,4 \%$ ) e do bíceps femoral (PRÉ 45,0 $\pm 5,4 \%$; PÓS $36,1 \pm 10,4 \%$ ) na subida na rampa. Na descida ocorreu aumento na velocidade da marcha (PRÉ 0,95 $\pm 0,18 \mathrm{~m} \cdot \mathrm{s}^{-1}$; PÓS 1,01 $\pm 0,22 \mathrm{~m} \cdot \mathrm{s}^{-1}$ ) e tamanho do passo (PRÉ 1,02 $\pm 0,15 \mathrm{~m}$; PóS 1,08 $\pm 0,18 \mathrm{~m}$ ). Conclusão: Os exercícios utilizados foram capazes de alterar de forma significativa os parâmetros avaliados, permitindo uma marcha mais veloz e reduzindo a ativação muscular. Estudos longitudinais são necessários para que os resultados aqui encontrados de forma temporária possam ser confirmados de forma permanente.

Palavras-chave: Marcha. Alongamento. Idosos. Eletromiografia.

\section{Abstract}

Introduction: The aging cause muscular modifications that have a negative influence on functional capacities of elderly on locomotion, in special during inclined surface transpose. Those changes generate decrease on gait performance, reducing the mobility and increasing the risk on execution of the task. The stretching exercises can reduce the influence of these degenerations, preserving the muscular capacities and the mobility. Objective: The aim of this study was evaluate the influence of acute effect of one session of stretching exercise on elderly gait during locomotion on sloped surface. Materials and methods: Twelve elderly women, healthy and independent, were walking up and down on a ramp with $10 \%$ of inclination. The kinematics (2D) and electromyography analysis was accomplished during one single experimental session, immediately before (PRE) and after (POS) a static stretching exercise on hip flexor muscles. Results: The stretching exercise was generate increases on range of motion (PRÉ $52.3 \pm 18.3^{\circ}$; PÓS $63.6 \pm 16.9^{\circ}$ ) and velocity (PRE $195.8 \pm 31.2^{\circ} / \mathrm{s}$; POS $241.8 \pm 29.8^{\circ} / \mathrm{s}$ ) of hip, as a reduction on time of activation of anterior tibialis (PRE TAFIN1 29.7 \pm 6.7\%; TAFIN2 100.0 \pm 0.0\%; POS TAFIN1 $23.3 \pm 7.4 \%$; TAFIN2 87.7 $\pm 3.4 \%$ ) and biceps femoris (PRE $45.0 \pm 5.4 \%$; POS 36,1 $\pm 10,4 \%$ ) on walking up. On walking down occur increases on gait velocity (PRE $0.95 \pm 0.18 \mathrm{~m} \cdot \mathrm{s}^{-1}$; POS $1.01 \pm 0.22 \mathrm{~m} \cdot \mathrm{s}^{-1}$ ) and step length (PRE $1.02 \pm 0.15 \mathrm{~m}$; POS $1.08 \pm 0.18 \mathrm{~m}$ ). Conclusion: The exercises were able to alter significantly the evaluates parameters, allowing a fast gait with reductions on muscle activation levels. Longitudinal studies are necessary to confirm chronically the temporary effects found on this study.

Keywords: Gait. Stretching. Elderly. Electromyography.

\section{Introdução}

Algumas alterações no padrão dinâmico da marcha decorrem de degenerações associadas ao processo de envelhecimento e modificam as propriedades visco-elásticas musculares $(1,2,3)$. A redução da amplitude articular da extensão do quadril durante a marcha causa alterações mecânicas em todo o membro inferior e leva à diminuição do tamanho do passo, da velocidade de deslocamento e da estabilidade da tarefa (4). Tais alterações potencializam a ocorrência de acidentes e aumentam o risco de quedas entre idosos (5).

Além das degenerações naturais do envelhecimento, a falta de exercícios regulares também pode influenciar as propriedades mecânicas do complexo músculo-tendão e a capacidade muscular de produzir força (6).
Exercícios de alongamento, às vezes associados a outros tipos de atividades, permitem aumento e manutenção da amplitude articular, pois alteram as características visco-elásticas da musculatura e modificam seu comportamento em atividades dinâmicas $(7,8)$.

Vários estudos tentaram identificar a influência de programas de exercícios de alongamento nas características visco-elásticas da musculatura $(9,10$, $11,12)$ ou na amplitude articular $(13,14,15)$. Alguns deles demonstram que exercícios de alongamento aplicados de forma aguda e crônica têm sido eficazes para modificar as propriedades musculares e a marcha em terreno plano $(16,17)$. Cristopoliski et al. (16) identificaram aumento do tamanho do passo, da velocidade de deslocamento e redução do período de apoio na marcha de idosos após quatro semanas de 
realização de exercícios de alongamento. Os autores relacionaram as modificações encontradas à redução do risco de quedas durante a marcha. Rodacki et al. (17) encontraram alterações na marcha de idosos após a realização de uma única sessão de alongamentos. Os resultados encontrados indicam que os efeitos agudos dos exercícios são similares aos que se manifestam de forma crônica, e podem ser utilizados para se identificar as mudanças esperadas na marcha após a realização de programas de aumento da flexibilidade.

Os efeitos agudos do alongamento podem modificar a marcha de idosos durante a subida e descida de rampas. Durante a transposição de superfícies inclinadas, existe maior demanda muscular $(18,19)$ e amplitudes articulares (20). 0 deslocamento na rampa é influenciado negativamente pela redução da capacidade contrátil (21) e da flexibilidade $(2,5)$ resultantes do processo de envelhecimento. Assim, as modificações agudas causadas pelo alongamento podem melhorar o desempenho da marcha na rampa e indicar quais mudanças seriam causadas na marcha de idosos em superfície inclinada, contribuindo com a adoção de um programa contínuo desse tipo de exercício.

A organização temporal da atividade muscular durante a marcha não é afetada pelo envelhecimento. Em um estudo conduzido por Monaco et al. (22), não foram verificadas diferenças nas ativações musculares em razão do aumento da idade. Entretanto, a marcha de idosos é influenciada pela demanda ambiental imposta durante a locomoção. 0 deslocamento em superfícies inclinadas pode causar modificações na organização da atividade muscular (cronometria), dificultar o controle e influenciar o aumento do risco de quedas durante a marcha.

Dessa maneira, é relevante observar as alterações influenciadas pelo efeito agudo do alongamento nos parâmetros cinemáticos e eletromiográficos da marcha de idosas. Tais modificações podem reduzir as limitações encontradas na marcha dessa população e conduzir o desenvolvimento de programas de exercícios mais efetivos para melhorar a qualidade de vida e a mobilidade desses indivíduos.

\section{Materiais e métodos}

Doze idosas $(71,07 \pm 7,0$ anos; $1,51 \pm 0,1 \mathrm{~m}$; $68,53 \pm 14,0 \mathrm{~kg}$ ), contatadas em diversos centros de convivência da terceira idade de Curitiba e Região
Metropolitana, e que voluntariamente se dispuseram a participar do presente estudo, fizeram parte de um único grupo experimental. Todos os indivíduos viviam normalmente na comunidade e realizavam suas atividades diárias sem auxílio externo de pessoas ou equipamentos. Além disso, não possuíam histórico de fraturas, cirurgias articulares, lombalgias ou outros problemas clínicos que pudessem interferir no padrão da marcha nos seis meses que precederam o início do estudo. Não foram incluídos indivíduos do sexo masculino em função de diferenças visco-elásticas entre os gêneros (23), o que poderia mascarar a influência da sessão de exercícios. Não foi realizada avaliação clínica prévia em razão dos critérios de seleção dos sujeitos e da natureza da atividade a ser realizada. Todas as participantes assinaram um termo de consentimento para a participação no estudo. 0 presente trabalho foi aprovado pelo comitê de ética do Setor de Ciências da Saúde da Universidade Federal do Paraná sob o número 01817012.6.0000.0102.

A marcha foi analisada imediatamente antes (PRÉ) e após (PÓS) os exercícios de alongamento, em uma única sessão. A avaliação da marcha na rampa ocorreu sobre uma superfície de madeira $(5,0 \times 1,0 \mathrm{~m})$ inclinada a $10 \%$ e revestida com um tapete emborrachado, para que a tarefa pudesse ser executada sem o uso de calçado. Ao final da rampa, foi colocado um patamar de 1,0 x 1,0 m, espaço esse utilizado para o retorno. A inclinação foi estabelecida dentro dos limites estabelecidos pela Associação Brasileira de Normas Técnicas (24) para a construção de rampas.

Cada participante realizou dez percursos em cada condição experimental (PRÉ e PÓS). Foram utilizados para a análise a média agrupada dos três primeiros ciclos válidos. Um ciclo foi considerado válido quando realizado sobre a rampa, onde os marcadores corporais foram capturados e o sinal elétrico muscular corretamente processado, permitindo a completa reconstrução do movimento. A velocidade de deslocamento não foi controlada e os participantes foram orientados a caminhar normalmente. Os dados foram normalizados em função da duração do ciclo da marcha ( $0 \%$ a $100 \%$, de $0,5 \%$ em $0,5 \%)$.

Foram fixados ao sujeito marcadores esféricos (25 $\mathrm{mm}$ ) em ambos os membros inferiores (espinhas ilíacas ântero-superiores, sacro, trocânter maior do fêmur, epicôndilo lateral do fêmur, maléolo lateral da fíbula, aspecto posterior do calcâneo e articulação metatarso-falangeal do segundo dedo). Entretanto, apenas o membro inferior direito foi avaliado. Esses marcadores foram 
utilizados para que um modelo biomecânico (2D) fosse criado para representar o movimento. A metodologia e o modelo escolhido são similares aos utilizado sem estudos anteriores $(2,5,16,17,25,26)$.

A análise cinemática foi realizada com uma câmera digital (EX-FH20, Cassio, Japão), com frequência de 200 $\mathrm{Hz}$ e shutter speed em 1/500 s. A câmera foi ajustada perpendicularmente ao movimento, na metade da distância entre o início e o fim da superfície inclinada da rampa. Os vídeos tiveram uma redução da frequência para $60 \mathrm{~Hz}$ durante a edição (VirtualDub, v. 1.9.7, Avery Lee, USA) e as imagens foram digitalizadas em um software específico (SkillSpector, v.1.0b, GeeWare, USA).

Para a avaliação da eletromiografia (EMG) foi utilizado um sistema de coleta de dados (Trigno Wireless, Delsys, USA), composto por seis eletrodos ativos. Após a identificação dos pontos de fixação dos eletrodos, foi realizada a limpeza e tricotomia da região. 0 posicionamento seguiu as orientações propostas pelo SENIAM (Surface ElectroMyoGraphy for the Non-Invasive Assessment of Muscles, disponível em www.seniam.org). A frequência de amostragem da EMG foi de $2000 \mathrm{~Hz}$, banda de frequência de sinal entre 20 a $450 \mathrm{~Hz}$, e ganho de amplificação total de $910 \pm 5 \%$. A EMG foi normalizada em razão do maior sinal (100\%) encontrado durante a marcha. A atividade elétrica mínima foi identificada e o músculo considerado ativo quando o sinal eletromiográfico foi superior à média da atividade elétrica mínima somada a dois desvios padrões.

Os vídeos coletados foram sincronizados com o eletromiógrafo a partir do sinal do acelerômetro acoplado ao eletrodo do calcâneo do membro avaliado. A mudança de direção da aceleração no eixo vertical indicou o contato do pé com o solo no início e no final do ciclo.

Entre os parâmetros cinemáticos, foram avaliados o tamanho do passo (TPAS), a velocidade da marcha (VEL), cadência (CAD), tempo total do ciclo (TTC), tempo de apoio (TA), tempo de oscilação (TO), velocidade de contato do pé (VELC), pico de velocidade de flexão do quadril (VELQUA), amplitude de movimento do quadril (AMPQUA), do joelho (AMPJOE) e do tornozelo (AMPTOR). Entre os parâmetros eletromiográficos, os instantes (\% do ciclo) de início (INI) e final (FIN) das atividades dos músculos tibial anterior (TAFIN1, TAINI e TAFIN2), gastrocnêmio lateral (GLFIN), vasto lateral (VLFIN1, VLINI e VLFIN2), bíceps femoral (BFFIN e BFINI) e glúteo médio (GMFIN) foram avaliados.
Todas as participantes selecionadas realizaram apenas uma sessão de exercícios. 0 protocolo consistiu em um exercício para alongamento da musculatura flexora do quadril, uni e bi-articular, aplicados em ambos os membros inferiores. Utilizou-se para o protocolo de alongamento a metodologia estática (27). 0 tempo de intervalo entre o término da sessão de exercícios e o início da filmagem da condição PÓS foi controlado e não foi superior a 30 segundos.

As idosas foram posicionadas em decúbito dorsal, com ambos os membros inferiores colocados em paralelo e suspensos para fora da maca. 0 quadril do membro não alongado foi flexionado em aproximadamente $125^{\circ} .0$ exercício foi aplicado por um segundo instrutor no membro suspenso. Realizou-se um deslocamento da coxa no sentido do solo (extensão do quadril) juntamente com uma flexão do joelho até o ponto em que o sujeito relatasse um pequeno desconforto muscular. Essa posição de extensão do quadril e flexão do joelho foi sustentada por 60 segundos. Após esse período, o procedimento foi aplicado no membro contralateral (alternadamente, 4 x 60 segundos em cada membro).

0 teste de Kolmogorov-Smirnov confirmou a normalidade dos dados. 0 teste de $t$-student para variáveis paramétricas foi aplicado para se identificar diferenças entre as condições PRÉ e PÓS. Os testes estatísticos tiveram nível de significância de $\mathrm{p} \leq 0,05$ (Statistica, versão 7.0).

\section{Resultados}

Durante os procedimentos, nenhuma participante relatou qualquer tipo de desconforto e todas cumpriram de maneira satisfatória o protocolo proposto. Não foram realizadas medições que quantificassem o efeito dos exercícios na amplitude articular, entretanto, foi calculado o coeficiente de variação $(28,29)$ para as amplitudes angulares. 0 coeficiente de variação (CV) representa a variabilidade média do parâmetro analisado durante a marcha. Em terreno plano, CV menores que 15\% representam grande consistência na execução do movimento (29). 0 CV é calculado dividindo-se o desvio padrão médio ao longo do ciclo pela média dos valores encontrados para a mesma variável.

Dessa forma, foi possível identificar a média da variabilidade intra-sujeitos e atribuir as modificações encontradas apenas ao protocolo de exercícios. A Tabela 1 resume os principais resultados encontrados nas variáveis cinemáticas. 
Não foram encontradas diferenças $(p>0,05)$ entre as condições PRÉ e PÓS nas variáveis de organização temporal do ciclo da marcha (TA, TO e TTC) durante as condições testadas, bem como a VELC e a CAD permaneceram sem mudanças ( $p>0,05)$. Entretanto, durante a descida, observou-se um aumento significativo $(\mathrm{p} \leq 0,05)$ do TPAS e da VEL entre as condições PRÉ e PÓS exercícios. No quadril, ocorreu um aumento $(\mathrm{p} \leq 0,05)$ da AMPQUA na subida entre as condições experimentais, e o músculo tibial anterior apresentou uma antecipação $(\mathrm{p} \leq 0,05)$ no instante de TAFIN1. Identificou-se também uma antecipação $(p \leq 0,05)$ no TAFIN2, que, na condição PRÉ, prosseguia até final do ciclo e, na condição PÓS, encerrou-se em $87,7 \pm$ $3,4 \%$ da atividade. 0 músculo bíceps femoral apresentou uma redução $(\mathrm{p} \leq 0,05)$ do BFFIN durante a subida da rampa. Não foram identificadas mudanças $(\mathrm{p}>0,05)$ na cronometria muscular durante a descida. A Tabela 2 apresenta os valores referentes às variáveis eletromiográficas (EMG) analisadas. As Figuras 1 e 2 apresentam o comportamento médio da ativação elétrica muscular (RMS) dos indivíduos durante marcha e a Figura 3 a cronometria de ativação muscular.

Tabela 1 - Variáveis cinemáticas durante a subida (SUB) e descida (DES) da rampa, antes (PRÉ) e após (PÓS) os exercícios

\begin{tabular}{|c|c|c|c|c|c|}
\hline & SUB PRÉ & SUB PÓS & & SUB PRÉ & SUB PÓS \\
\hline TPAS (m) & $1,20 \pm 0,1$ & $1,22 \pm 0,1$ & TA (\%) & $66,80 \pm 3,0$ & $66,25 \pm 2,6$ \\
\hline VEL $\left(m \cdot s^{-1}\right)$ & $1,00 \pm 0,1$ & $1,04 \pm 0,1$ & TO (\%) & $33,20 \pm 3,0$ & $33,75 \pm 2,6$ \\
\hline $\operatorname{VELC}\left(m \cdot s^{-1}\right)$ & $0,59 \pm 0,4$ & $0,80 \pm 0,4$ & AMPQUA $\left({ }^{\circ}\right)$ & $52,3 \pm 18,3$ & $63,6 \pm 16,9^{x}$ \\
\hline TTC (s) & $1,22 \pm 0,1$ & $1,19 \pm 0,1$ & AMPJOE $\left({ }^{\circ}\right)$ & $48,1 \pm 7,8$ & $48,7 \pm 6,2$ \\
\hline \multirow[t]{3}{*}{ CAD (passos/s) } & $49,85 \pm 5,3$ & $50,86 \pm 5,7$ & AMPTOR $\left(^{\circ}\right)$ & $28,3 \pm 11,2$ & $29,6 \pm 9,6$ \\
\hline & & & VELQUA $(\% / s)$ & $195,8 \pm 31,2$ & $241,8 \pm 29,8^{\star}$ \\
\hline & DES PRÉ & DES PÓS & & DES PRÉ & DES PÓS \\
\hline TPAS (m) & $1,02 \pm 0,15$ & $1,08 \pm 0,18^{*}$ & TA (\%) & $64,71 \pm 2,44$ & $64,59 \pm 3,00$ \\
\hline VEL $\left(m \cdot s^{-1}\right)$ & $0,95 \pm 0,18$ & $1,01 \pm 0,22^{*}$ & TO (\%) & $35,29 \pm 2,44$ & $35,41 \pm 3,00$ \\
\hline $\operatorname{VELC}\left(m \cdot s^{-1}\right)$ & $0,44 \pm 0,29$ & $0,62 \pm 0,44$ & AMPQUA $\left({ }^{\circ}\right)$ & $35,5 \pm 8,44$ & $0,6 \pm 10,3$ \\
\hline TTC (s) & $1,10 \pm 0,10$ & $1,09 \pm 0,11$ & AMPJOE $\left({ }^{\circ}\right)$ & $61,2 \pm 6,36$ & $3,2 \pm 5,3$ \\
\hline \multirow[t]{2}{*}{ CAD (passos/s) } & $55,14 \pm 5,13$ & $55,70 \pm 5,44$ & AMPTOR $\left({ }^{\circ}\right)$ & $20,6 \pm 4,42$ & $1,0 \pm 5,6$ \\
\hline & & & VELQUA $(\% / s)$ & $140,7 \pm 22,7$ & $188,8 \pm 27,1$ \\
\hline
\end{tabular}

Nota: $\left(^{*}\right)=$ diferença significativa entre as condições PRÉ e PÓS; foi utilizado $p \leq 0,05$.

Tabela 2 - Variáveis eletromiográficas durante a subida (SUB) e descida (DES) da rampa antes (PRÉ) e após (PÓS) os exercícios

\begin{tabular}{|c|c|c|c|c|c|}
\hline & SUB PRÉ & SUB PÓS & & SUB PRÉ & SUB PÓS \\
\hline TAFIN1 (\%) & $29,7 \pm 6,7$ & $23,3 \pm 7,4^{*}$ & VLFIN2 (\%) & $69,7 \pm 6,8$ & $65,7 \pm 7,5$ \\
\hline TAINI (\%) & $59,3 \pm 2,2$ & $58,5 \pm 2,8$ & GLFIN (\%) & $32,7 \pm 10,7$ & $33,1 \pm 9,0$ \\
\hline TAFIN2 (\%) & $100,0 \pm 0,0$ & $87,7 \pm 3,4^{*}$ & BFFIN (\%) & $45,0 \pm 5,4$ & $36,1 \pm 10,4$ \\
\hline VLFIN1 (\%) & $18,5 \pm 5,6$ & $17,6 \pm 2,9$ & BFINI (\%) & $86,2 \pm 2,4$ & $83,5 \pm 5,9$ \\
\hline \multirow[t]{2}{*}{ VLINI (\%) } & $33,5 \pm 3,2$ & $29,8 \pm 5,2$ & GMFIN (\%) & $47,2 \pm 14,0$ & $0,6 \pm 2,7$ \\
\hline & DES PRÉ & DES PÓS & & DES PRÉ & DES PÓS \\
\hline TAFIN1 (\%) & $28,7 \pm 6,5$ & $31,2 \pm 6,4$ & VLFIN2 (\%) & $89,8 \pm 2,1$ & $89,0 \pm 6,9$ \\
\hline TAINI (\%) & $57,4 \pm 4,5$ & $59,7 \pm 5,0$ & GLFIN (\%) & $34,2 \pm 12,9$ & $38,2 \pm 11,5$ \\
\hline TAFIN2 (\%) & $87,3 \pm 3,2$ & $88,8 \pm 4,4$ & BFFIN (\%) & $25,0 \pm 9,9$ & $26,7 \pm 5,8$ \\
\hline VLFIN1 (\%) & $40,3 \pm 6,1$ & $38,4 \pm 10,2$ & BFINI (\%) & $76,9 \pm 11,7$ & $80,2 \pm 4,9$ \\
\hline VLINI (\%) & $69,1 \pm 1,0$ & $74,9 \pm 11,1$ & BFINI (\%) & $76,9 \pm 11,7$ & $40,7 \pm 13,4$ \\
\hline
\end{tabular}

Nota: $\left({ }^{*}\right)=$ diferença significativa entre as condições PRÉ e PÓS; foi utilizado $p \leq 0,05$. 


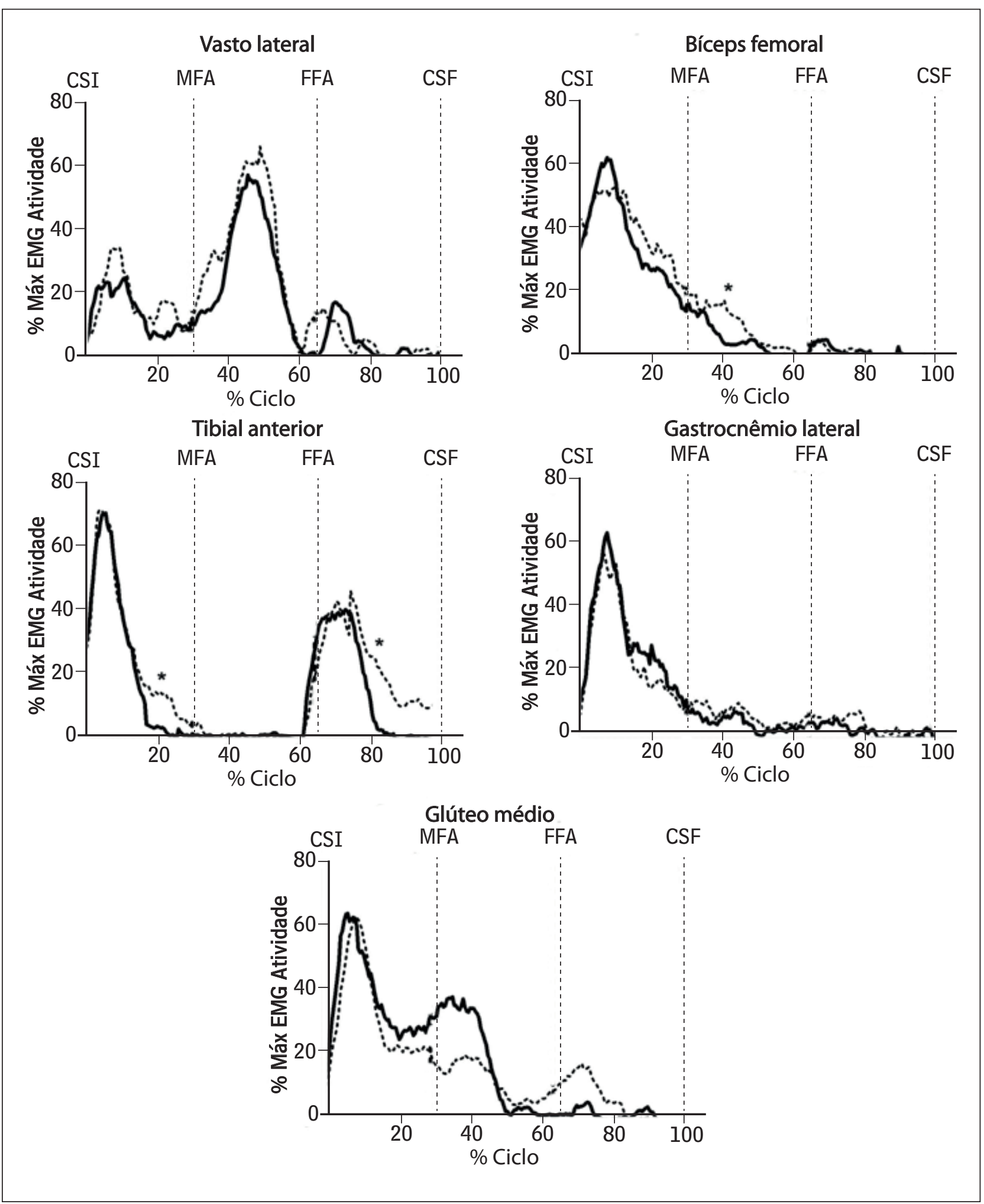

Figura 1 - RMS da EMG durante a subida

Nota: PRÉ: linha pontilhada; PÓS: linha cheia; CSI: contato com o solo inicial; MFA: meio da fase de apoio; FFA: final da fase de apoio; CSF: contato com o solo final. $\left(^{*}\right)=$ diferença entre PRÉ e PÓS $(p \leq 0,05)$. 


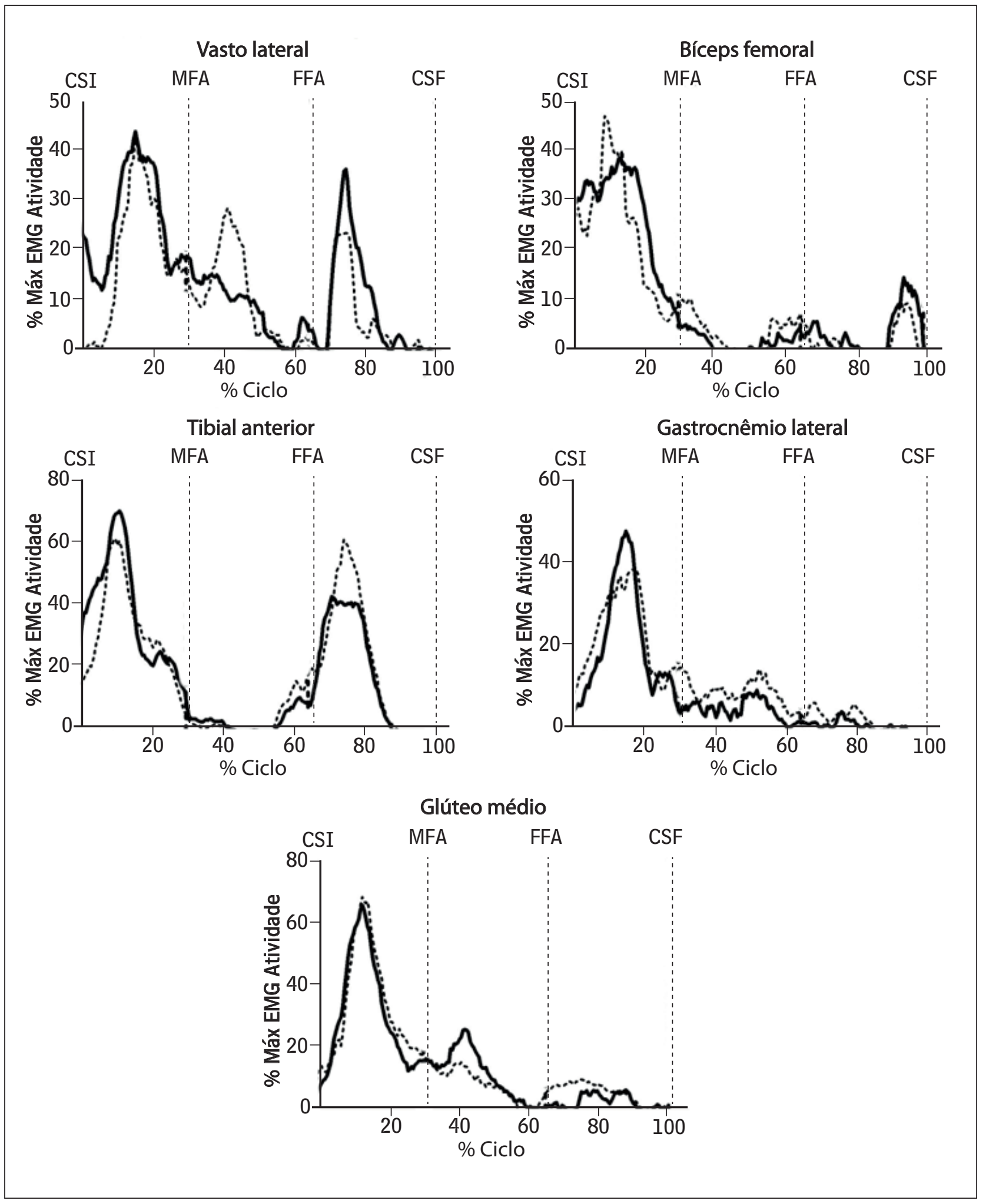

Figura 2 - RMS da EMG durante a descida

Nota: PRÉ: linha pontilhada; PÓS: linha cheia; CSI: contato com o solo inicial; MFA: meio da fase de apoio; FFA: final da fase de apoio; CSF: contato com o solo final. 


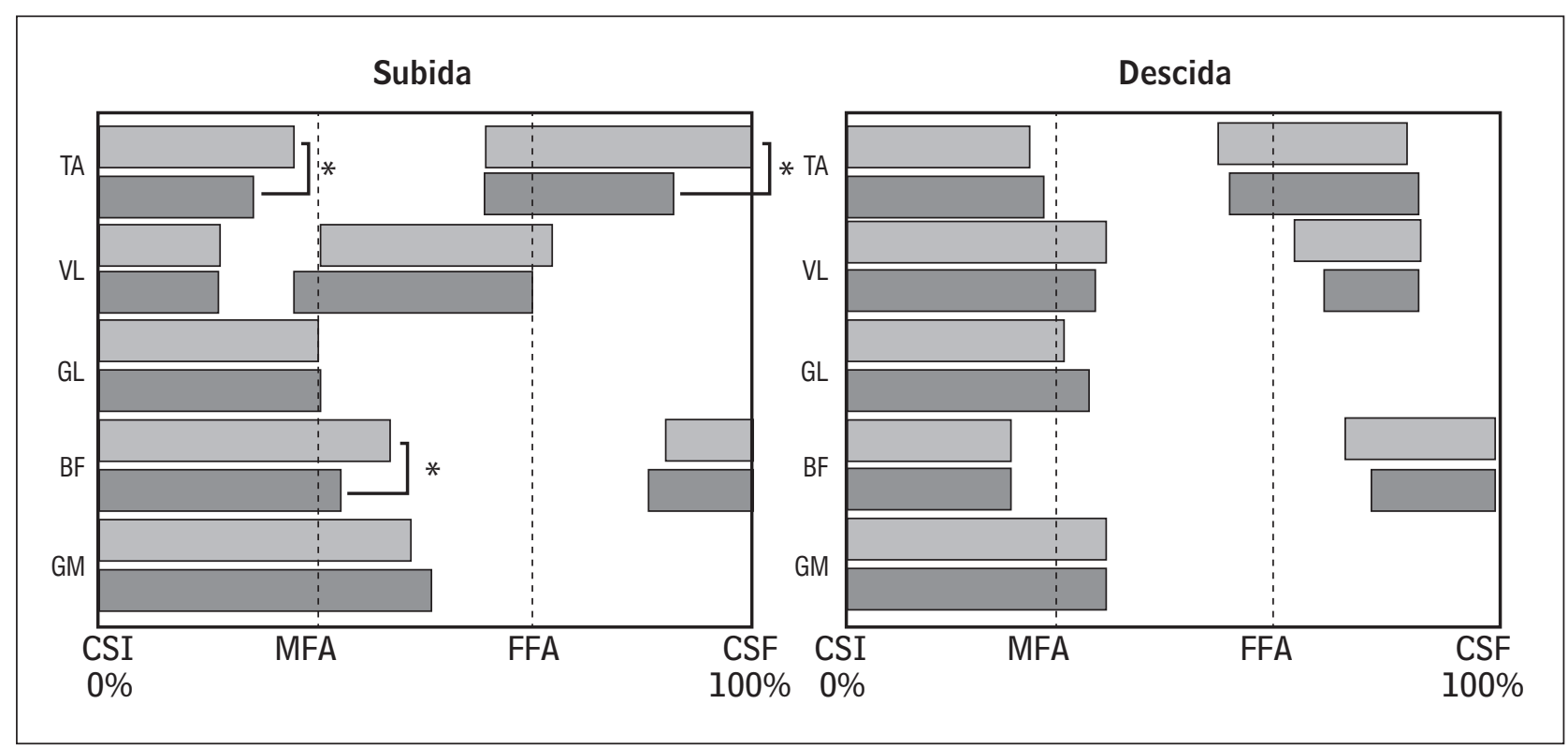

Figura 3 - Cronometria muscular na subida e na descida

Nota: As barras identificam o período de atividade muscular; PRÉ: barras claras; PÓs: barras escuras; CSI: contato com o solo inicial; MFA: meio da fase de apoio; FFA: fase final de apoio; CSF: contato com o solo final. TA = tibial anterior; VL = vasto lateral; $G L=$ gastrocnêmio lateral; $\mathrm{BF}=$ bíceps femoral; $\mathrm{GM}=$ glúteo máximo; $\left({ }^{*}\right)$ = diferença significativa $(\mathrm{p} \leq 0,05)$.

\section{Discussão}

0 presente estudo teve como objetivo verificar as alterações agudas nos parâmetros cinemáticos e eletromiográficos da marcha de idosas em terreno inclinado após a realização de uma sessão de exercícios de alongamento.

As médias dos coeficientes de variação das variáveis angulares (intrassujeito) foram de aproximadamente $4,2 \%$ no PRÉ e 5,5\% no PÓS teste e indicam que todas as participantes foram capazes de realizar a marcha de maneira consistente. São considerados normais para o coeficiente de variação intrassujeito, em variáveis angulares na marcha (2D), valores em torno de $15 \%(28,29)$. Essa baixa variabilidade pode ter sido influenciada pelos critérios utilizados na seleção dos participantes, que admitia apenas idosas saudáveis e relativamente ativas. Dessa forma, as modificações encontradas na marcha foram atribuídas à sessão de exercícios de alongamento proposta, similar a protocolos previamente utilizados (17) e considerada adequada para os objetivos experimentais.

0 estudo de Taylor et al. (30) demonstrou que os ganhos elásticos do complexo músculo-tendíneo são conseguidos até a quarta repetição dos exercícios de alongamento. Outros estudos $(31,32,33)$ têm demonstrado que exercícios de alongamento com tempo de aplicação igual ou superior a 60 segundos são eficazes para causarem modificações nas características visco-elásticas do complexo músculo-tendão de indivíduos similares aos participantes deste estudo. Estudos que buscaram analisar os efeitos dos exercícios de alongamento $(27,31,32,33,34)$ têm utilizado protocolos similares. 0 estudo realizado por Spernoga et al. (14) analisou as características elásticas do tecido muscular em função de exercícios de alongamento e demonstrou que seus efeitos ainda eram significativos 6 minutos após o encerramento da sessão de exercícios. Desse modo a realização de exercícios de alongamentos estáticos, compostos por quatro séries de 60 segundos de duração, foi suficiente para causar alterações elásticas na musculatura manipulada e influenciar mudanças em alguns aspectos da marcha das participantes.

Quando se objetiva analisar a capacidade de locomoção, o terreno inclinado oferece um desafio maior do que a transposição de superfícies planas, o que tem sido evidenciado pela maior frequência de acidentes (35). Assim, a utilização da rampa para a avaliação dos efeitos agudos dos exercícios, sobre os parâmetros cinemáticos e eletromiográficos da marcha, parece ser mais atrativa do que a transposição de terrenos planos. 
As idosas apresentaram um maior TA e um menor TO em comparação com os resultados encontrados para jovens ( $24 \pm 3$ anos), apesar de um TTC similar (36). O TPAS, a CAD e a VEL dos idosos na condição PRÉ, na subida e descida da rampa, também foram menores em comparação a jovens durante a marcha na mesma inclinação (20). Não foram encontrados estudos com população e condições experimentais similares.

As diferenças encontradas entre a organização do passo do idoso e do jovem na rampa são percebidas também no deslocamento em terreno plano, e refletem a necessidade de maior TA para a estabilização e execução do próximo passo (37). A redução do TPAS e VEL e o aumento do TA são influência de uma menor capacidade de deslocamento. A redução do TPAS e da VEL está diretamente ligada à redução das capacidades elásticas e contráteis do complexo músculo-tendíneo em razão das degenerações do envelhecimento (38). Essas alterações levam à realização de um passo menos estável e mais suscetível a acidentes.

Durante a subida, os parâmetros da marcha referentes à organização espaço-temporal do ciclo (TTC, TA, TO e CAD), não sofreram modificações após os exercícios. Na descida encontrou-se um aumento do TPAS e da VEL após a realização dos exercícios de alongamento. $\mathrm{O}$ aumento do TPAS, e por consequência da VEL, é resultado da redução da limitação mecânica causada pelo grupo muscular manipulado, aumentando a amplitude articular (5). Nenhuma mudança foi encontrada nas amplitudes e picos angulares da marcha durante a descida. Entretanto, a manipulação dos músculos exercitados pode ter reduzido a complacência muscular $(1,39)$ e influenciado positivamente a marcha. Apesar de não terem sido verificadas mudanças ao redor da articulação exercitada, modificações na porção mais distal do membro foram geradas. Essas modificações permitiram a execução de um TPAS de maior amplitude, o que aumentou a velocidade sem reduzir a consistência da marcha durante a locomoção.

Durante a descida, é necessário realizar contrações excêntricas com maior intensidade. No envelhecimento a capacidade contrátil vai diminuindo, entretanto, a força excêntrica é a que sofre as menores reduções durante esse processo (40). Sendo assim, essa redução mais lenta da força excêntrica durante o envelhecimento permitiu aos idosos realizar e controlar uma descida mais veloz.

A velocidade de deslocamento tem sido descrita como um dos melhores indicadores de segurança na marcha. Incrementos em torno de $0,1 \mathrm{~m} \cdot \mathrm{s}^{-1}$ na velocidade de deslocamento de idosos estão diretamente associados com a redução do risco de acidentes durante a transposição de terrenos planos (41). Acredita-se que, de forma similar ao que acontece em terreno plano, incrementos na velocidade de deslocamento na rampa sejam reflexo de maior capacidade de controle. Sendo assim, os exercícios aplicados foram capazes de diminuir a resistência imposta pelo músculo manipulado, permitindo uma descida mais veloz e menos suscetível a acidentes.

Na subida, os exercícios de alongamento causaram um aumento na MAXQUA e na AMPQUA. Esse achado corrobora os resultados de estudos anteriores que investigaram os efeitos de exercícios de alongamento na manutenção de uma adequada capacidade flexível dos músculos flexores do quadril (uni e bi-articulares) para a execução mais eficiente da marcha $(2,5,42)$. A redução da capacidade de extensão dessa musculatura durante atividades dinâmicas tem sido descrita como um componente que pode aumentar a probabilidade de acidentes (1), principalmente em deslocamentos sobre superfícies mais desafiadoras e em populações que têm reduções da força, equilíbrio e perdas gradativas da flexibilidade (39).

0 aumento temporário da elasticidade induzido pelos exercícios de alongamento permitiu uma maior EXTQUA assim como um aumento da velocidade de flexão do quadril (VELQUA) durante a oscilação na subida da rampa. Esse aumento da velocidade de flexão pode ser atribuído a aumentos da força elástica do complexo músculo-tendão em resposta aos exercícios de alongamento. Cavagna (43) relata que os tecidos musculares e tendíneos mais complacentes são mais eficientes para acumular energia elástica e utilizá-la durante atividades de alongamento-encurtamento. Resultados similares foram encontrados em estudo que avaliou a característica elástica dos tendões e a performance do salto de adultos (44). Os autores encontraram uma correlação direta entre a flexibilidade do tendão, a energia elástica acumulada e a altura do salto.

O aumento da EXTQUA e da AMPQUA foram causados pela modificação temporária da elasticidade 
dos flexores do quadril. Esse aumento da elasticidade induziu o aumento da VELQUA e um maior pico de flexão do joelho (FLXJOE) durante a oscilação. A flexão do joelho durante a marcha é causada pela rápida flexão do quadril e da coxa (4) durante a oscilação e não pela ação dos músculos flexores dessa articulação, que não apresentam atividade contrátil nessa fase do movimento (45). 0 aumento da VELQUA do membro oscilante causou uma oscilação com maior velocidade. Esse movimento mais veloz do membro causa um aumento na contribuição da oscilação para o desenvolvimento da marcha ascendente. Dessa forma, o tempo de contração do BF necessário para estender o membro contralateral (BFFIN) e auxiliar no impulso para o passo foi reduzido. A maior FLXJOE reduziu a distância de alcance da perna lançada e compensou o aumento que a maior EXTQUA poderia gerar no TPAS. 0 aumento da FLXJOE permitiu um contato do pé com o solo em uma posição menos inclinada em relação à rampa, o que provocou um encerramento mais precoce do TAFIN1.

Durante a fase de oscilação é necessária a contração do tibial anterior para que o ante-pé possa se afastar do solo e permitir o próximo passo. $\mathrm{O}$ aumento da FLXJOE também reduziu o tamanho total do membro oscilante e exigiu um menor tempo de ativação do tibial anterior durante essa fase, o que antecipou o instante do TAFIN2. Winter (29) relata que o afastamento do pé em relação ao solo é geralmente relacionado a alterações no ângulo de dorsiflexão do tornozelo. Entretanto, resultados prévios indicam que esse afastamento é influenciado por mínimas mudanças ocorridas ao redor da articulação do joelho durante o pico de flexão. Mesmo alterações de pequenas magnitudes $\left(\sim 1,35^{\circ}\right)$ na flexão do joelho são capazes de contribuir de forma significativa para o aumento do afastamento do pé em relação ao solo (46). Os resultados confirmam tal argumento, onde o aumento da FLXJOE foi capaz de reduzir o tamanho total do membro oscilante, de forma a gerar um encerramento mais precoce da ativação do tibial anterior, necessária para que houvesse um afastamento da porção distal do pé em relação ao solo.

O mecanismo de aumento da EXTQUA e FLXJOE parece ser fundamental para que exista a manutenção do TPAS na subida. Um aumento do TPAS durante a subida pode aumentar a intensidade das contrações musculares, gerando maior esforço e gasto energético ao idoso. Durante a marcha em terreno plano, o aumento do tamanho do passo em $40 \%$ gera um aumento do custo energético em $26 \%$ quando comparado ao passo realizado com amplitude confortável (47). Provavelmente, durante a marcha ascendente, um maior custo energético pode ser encontrado com o aumento do TPAS.

Desse modo, as mudanças encontradas nos parâmetros da marcha avaliados, como o aumento da amplitude articular, o aumento da velocidade na descida e a redução do tempo de contração muscular na subida, acabaram influenciando um passo mais eficiente e reduzindo as limitações mecânicas causadas pela perda da elasticidade muscular.

\section{Conclusão}

O exercício de alongamento executado causou mudanças nas características dinâmicas da tarefa, tanto durante a subida quanto na descida da rampa. $\mathrm{O}$ aumento da velocidade da descida após os exercícios foi consequência da capacidade de execução de uma marcha mais estável, e dessa forma mais segura. Durante a subida, a redução das atividades musculares foram reflexo de um aumento do componente elástico muscular. São necessários futuros estudos para se verificar se os resultados aqui encontrados de forma aguda podem ser conseguidos de forma crônica após a realização de atividades sistematizadas.

\section{Referências}

1. Kerrigan DC, Xenopoulos-Oddsson A, Sullivan MJ, Lelas JJ, Riley P. Effect of a hip flexor-stretching program on gait in the elderly. Arch Phys Med Rehabil. 2003;84(1):1-6.

2. Kerrigan DC, Lee LW, Collins JJ, Riley PO, Lipsitz LA. Reduce hip extension during walking: healthy elderly and fallers versus young adults. Arch Phys Med Rehabil. 2001;82(1):26-30.

3. Alter MJ. Ciência da flexibilidade. 2. ed. Porto Alegre: Artmed;1999.

4. Viel E. A marcha humana, a corrida e o salto: biomecânica, investigações, normas e disfunções. Barueri: Manole; 2001. 
5. Kerrigan DC, Todd MK, Della Croce U, Lipsitz LA, Collins JJ. Biomechanical gait alterations independent of speed in the healthy elderly: evidence for specific limiting impairments. Arch Phys Med Rehabil. 1998;79(3):317-22.

6. Kubo K, Akima H, Kouzaki M, Ito M, Kawakami Y, Kanehisha H, et al. Changes in the elastic properties of tendon structures following 20 days bed-rest in humans. Euro J App Physiol. 2000;83(6):463-8.

7. Sartor CD, Watari R, Pássaro AC, Picon AP, Hasue RH, Sacco IC. Effects of a combined strengthening, stretching and functional training program versus usual-care on gait biomechanics and foot function for diabetic neuropathy: a randomized controlled trial. BMC Musculoskelet Disord. 2012;13:36.

8. Zakas A, Galazoulas C, Grammatikopoulou MG, Vergou A. Effects of stretching exercise during strength training in prepubertal, pubertal and adolescent boys. J Body Mov Therap. 2001;6(3):170-6.

9. Kubo K, Kanehisa H, Fukunaga T. Effect of stretching training on the viscoelastic properties of human tendon structures in vivo. J App Physiol. 2002;92(2):595-601.

10. Chan SP, Hong Y, Robinson PD. Flexibility and passive resistance of the hamstring of Young adults using two different static stretching protocols. Scand J Med Sci Sports. 2001;11(2):81-6.

11. Hunter DG, Coveney V, Spriggs J. Investigation into the effect of static stretching on the active stiffness and damping characteristics of the ankle joint plantar flexors. Phys Ther Sports. 2001;2(1):15-22.

12. Halbertsma JPK, Van Bolhuis AI, Göeken LNH. Sport stretching: effect on passive muscle stiffness of short hamstrings. Arch Phys Med Rehab. 1996;77(7):688-92.

13. Schuback B, Hooper J, Salisbury L. A comparison of a self-stretch incorporating proprioceptive neuromuscular facilitation components and a therapist-applied PNF-technique on hamstring flexibility. Physiotherapy. 2004;90(3):151-7.

14. Spernoga SG, Uhl TL, Arnold BL, Gansneder BM. Duration of maintained hamstring flexibility after onetime, modified hold-relax stretching protocol. J Athl Train. 2001;36(1):44-8.
15. Kirsch RF, Weiss PL, Dannenbaum RM, Kearney RE. Effect of maintained stretch on the range of motion of the human ankle joint. Clin Biomech. 1995;10(3):166-8.

16. Cristopoliski F, Barela JA, Leite N, Fowler NE, Rodacki ALF. Stretching exercise program improves gait in the elderly. Gerontology. 2009;55(6):614-20.

17. Rodacki ALF, Souza RM, Ugrinowitsch C, Cristopoliski F, Fowler N. Transient effect of stretching exercises on gait parameters of elderly women. Man Ther. 2008;14(2):167-72.

18. Wall-Scheffler CM, Chumanov E, Steudel-Numbers K, Heiderscheit B. Eletromyography activity across gait and incline: the impact of muscular activity on human morphology. Am J Phys Anthrop. 2010;143(4):601-11.

19. Minetti AE, Moia C, Roi GS, Susta DV, Ferretti G. Energy cost of walking and running at extreme uphill and downhill slopes. J App Physiol. 2002;93(3):1039-46.

20. McIntosh AS, Beatty KT, Dwan LN, Vickers DR. Gait dynamics on inclined walkway. J Biomech. 2006;39(13):2491-502.

21. Muscaritoli M, Lucia S, Molfino A, Cederholm T, Fanelli FR. Muscle atrophy in aging and chronic diseases: it is sarcopenia or cachexia? Intern Emerg Med. 2013;8(7):553-60.

22. Monaco V, Ghionzoli A, Micera S. Age-related modifications of muscle synergies and spinal cord activity during locomotion. J Neurophysiol. 2010;104(4):2092-102.

23. Kubo K, Kanehisa H, Fukunaga T. Gender diferences in the viscoelastic properties of tendo structures. Euro J Appl Physiol. 2003;88(6):520-6.

24. Associação Brasileira de Normas Técnicas. Norma brasileira NBR 9050: acessibilidade à edificações, mobiliário, espaços e equipamentos urbanos. Rio de Janeiro: ABNT; 2004.

25. Evans J, Zavarei K, Lelas JJ, Riley PO, Kerrigan DC. Reduce hip extension in the elderly: dynamic or postural? Arch Phys Med Rehabil. 2003;84(9):E15.

26. Kerrigan DC, Xenopoulos-Odsson A, Sullivan MJ, Lelas JJ, Riley PO. Effect of a hip flexor-stretching program on gait in the elderly. Arch Phys Med Rehabil. 2003;84(1):1-6. 
27. Feland JB, Myrer JW, Merrill RM. Acute changes in hamstring flexibility: PNF versus static stretch in senior athletes. Phys Ther Sport. 2001;2(4):186-193.

28. Kirtley C. Clinical analysis: theory and practice. Churchil Livingstone: Elsevier; 2006.

29. Winter DA. The biomechanics and motor control of human gait: normal, elderly and pathological. 2. ed. Waterloo: University of Waterloo Press;1991.

30. Taylor DC, Dalton JD, Seaber AV, Garrett WE. Viscoelastic properties of muscle-tendon units: the biomechanical effects of stretching. Am J Sports Med. 1990;18(3):300-9.

31. Zakas A, Balaska P, Grammatikopoulou MG, Zakas N, Vergou A. Acute effects of stretching duration of range of motion of elderly woman. J Bodyw Mov Ther. 2005;9(4):270-6.

32. Zakas A. The effect of stretching duration on the lower extremity flexibility of adolescent soccer players. J Bodyw Mov Ther. 2005;9(3):220-5.

33. Bandy WD, Irion JM, Briggler M. The effect of time and frequency of static stretching on flexibility of the hamstring muscles. Phys Ther. 1997;77(10):1090-6.

34. Feland JB, Myrer JW, Schulthies SS, Fellingham GW, Measom GW. The effect of duration of stretching of the hamstring muscle group for increasing range of motion in people aged 65 years or older. Phys Ther. 2001;81(5):1110-7.

35. Toscano G, Windau J. Fatal work injuries: results from the 1992 national census. Mon Larbor Rev. 1993;1993:39-48.

36. Lay AN, Hass CJ, Gregor RJ. The effects of sloped surfaces on locomotion: a kinematic and kinetic analysis. J Biomech. 2006;39(9):1621-8.

37. Prince F, Corriveau H, Hébert R, Winter DA. Gait in the elderly. Gait Posture. 1997;5(2):128-35.

38. Seene T, Kaasik P. Role of exercise therapy in prevention of decline in aging muscle function: glucocorticoid myopathy and unloading. J Aging Res. 2012;2012:172492.
39. Davis-Hammonds AL, Laudner KG, McCawS, McLoda TA. Acute lower extremity running kinematics after a hamstring stretch. J Athl Train. 2012;47(1):5-14.

40. Roig M, Macintyre DL, Eng JJ, Narici MV, Maganaris CN, Reid WD. Preservation of eccentric atrength in older adults: evidence, mechanisms and implications for training and rehabilitation. Exp Gerontol. 2010;45(6):400-9.

41. Blanco I, Verghese J, Lipton RB, Putterman C, Derby CA. Racial differences in gait velocity in an urban elderly cohort. J Am Geriatr Soc. 2012;60(5):922-6.

42. Watt JR, Jackson K, Franz JR, Dicharry J, Evans J, Kerrigan DC. Effect of a supervised hip flexor stretching program on gait in frail elderly patients. PM R. 2011;3(4):330-5.

43. Cavagna GA. Storage and utilization of elastic energy in skeletal muscle. Exerc Sports Sci Rev. 1977;5:89-129.

44. Kubo K, Kawakami Y, Fukunaga T. Influence of elastic properties of tendo structures on jump performance in humans. J App Physiol. 1999;87(6):2090-6.

45. Nymark JR, Balmer SJ, Melis EH, Lamaire ED, Millar S. Electromyographic and kinematic nondisabled gait differences at extremely slow overground and treadmill walking speeds. J Rehabil Res Dev. 2005;42(4):523-34.

46. Winter DA. Foot trajectory in human gait: a precise and multifactorial motor control task. Phys Ther. 1992;72(1):45-53.

47. Sawicki GS, Ferris DP. Powered ankle exoskeletons reveal the metabolic cost of plantar flexor mechanical work during walking with longer steps at constant step frequency. J Exp Biol. 2009;212(Pt 1):21-31.

Recebido: 11/06/2014

Received: 06/11/2014

Aprovado: 27/02/2015

Approved: 02/27/2015 